\title{
The Taming of the Screw: the natural and artificial development of $\beta$-propeller proteins
}

\author{
Bram Mylemans, Arnout R.D. Voet, Jeremy R.H. Tame
}

September 2020

\section{Contact}

\author{
Jeremy Tame \\ Protein Design Laboratory \\ Graduate School of Medical Life Science \\ Yokohama City University \\ Suehiro 1-7-29, Tsurumi, Yokohama 230-0045, Japan \\ tel:045-508-7228 fax:045-508-7366 \\ email: \\ jtame@yokohama-cu.ac.jp \\ arnout.voet@kuleuven.be \\ bram.mylemans@kuleuven.be
}

\section{Abstract}

Proteins are produced as unbranched polymers whose sequence is determined by information stored in linear fashion as DNA. Replication errors of genetic material accumulate, and tend to increase diversity of both the sequence and length of the proteins produced. Many proteins are found to possess repeated structural elements, which hint at ancient evolutionary origins and ongoing evolutionary processes. $\beta$ propeller proteins are a large family of such proteins, and a popular focus of structural analysis. This review highlights recent work to understand how they arose, and how they have developed into one of the most successful of all protein folds.

\section{Introduction}

Duplication and fusion events are relatively common forms of DNA copying error [1], and result in tandem sequence repeats at the protein level [2]. A recent extensive study reveals that more than $60 \%$ of eukaryotic proteins possess this feature, although most repeats are short, with fewer than 15 amino acid residues. Around $30 \%$ of duplicate insertions are larger repeats of whole domains [3]. Tandem repeat proteins (TRPs) have been classified into two different groups, open repeat proteins with distant N- and $\mathrm{C}$ - termini, and closed repeats with the chain (or domain) termini close together [4]. Examples of the first group include the ankyrin repeats and leucine-rich repeats (LRR) [5], while the TIM or $\alpha / \beta$-barrel is a common closed repeat fold [6].

The $\beta$-propeller proteins are the largest family of closed TRPs. The repeat units, or blades, are composed of four anti-parallel strands, and arranged around a central symmetry axis (Figure 1). While 


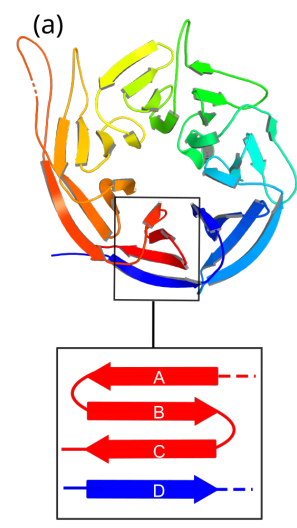

(b)
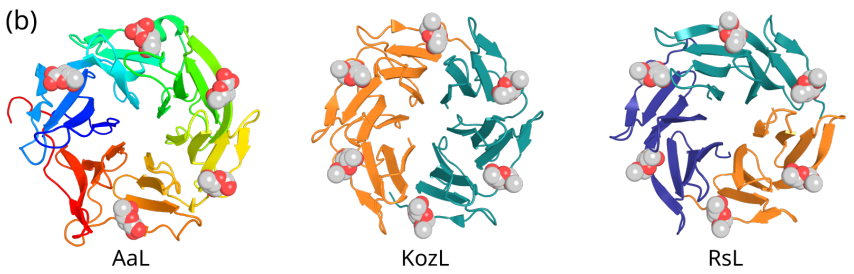

(c)
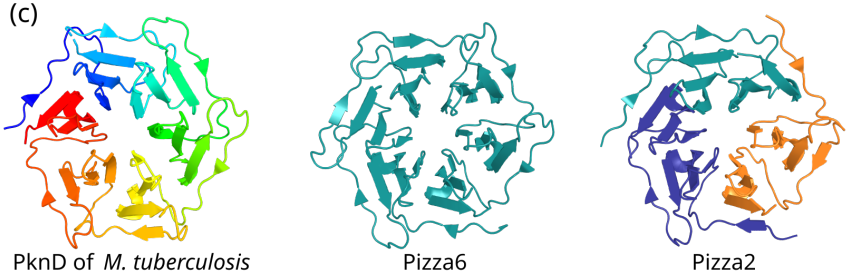

Figure 1: Monomeric and oligomeric forms of $\beta$-propeller. (a) The seven-bladed $\beta$-propeller protein WDR92 (PDB:3I2N)[15], coloured from blue to red ( $\mathrm{N}$ to $\mathrm{C}$ terminus) with a schematic inset showing an offset between the sequence and the supersecondary structure known as "velcro". (b) A monomeric lectin from $A$. aurantia $(\mathrm{AaL})$, coloured from $\mathrm{N}$ to $\mathrm{C}$ terminus. The homo-dimeric and homo-trimeric lectins KozL and RsL from K. zhangzhouensis and R. solanacearum, coloured by chain, with the ligands shown as space-filling models. (c) The PknD domain from Mycobacterium tuberculosis (PDB:1RWL), the template for the artificial, symmetrised Pizza6 protein. Expressing the two-bladed fragment Pizza2 yields a homo-trimeric propeller.

other closed repeat families have a fixed number of repeats, $\beta$-propellers vary in size. Six-, seven- and eight-bladed forms are most prevalent, but $\beta$-propellers exist with as few as four repeats [7] and as many as ten on a single polypeptide chain [8]. Small propellers often have ligand binding properties, and include many lectins known as PropLecs [9]. Larger variants tend to function as enzymes or structural proteins, while the largest $\beta$-propellers more generally participate in signalling pathways [10]. Although they all possess a similar structure, $\beta$-propellers show a large sequence diversity and can be subdivided into families defined by characteristic sequence motifs [11]. WD40 propellers have conserved neighbouring tryptophan and aspartic acid residues in each blade for example, while NHL propellers are named after three family members.

\section{$3 \quad \beta$-propeller features}

\subsection{Repeats}

Current evolutionary theory strongly supports the hypothesis that proteins have evolved by duplication and fusion of small peptides, a process whose "fossilised" remnants are most evident in repeat proteins [12]. Propeller proteins have been the focus of several analytical investigations into this process, for example by comparing exon/intron boundaries with structural features of repeat proteins [13]. A bioinformatics study by Lupas and colleagues in 2008 supported the notion that that the major families of propellers were amplified independently from single blades, and that this is an ongoing process [14]. They found that the different sub-families are closely related, irrespective of the number of blades present, suggesting a common (homologous) origin for most propeller proteins, if not all. The evolutionary pathway from onebladed fragments to complete monomeric propellers is predicted to involve homo-oligomeric intermediates, and the existence of natural propeller proteins of this type is strong evidence for evolution through duplication (Figure 1). Examples are rare but include a lectin from the bacterium Ralstonia solanacearum (PDB:2BT9) [16] and a related fucose binding lectin from Burkholderia ambifaria (PDB:3ZW0) [17], which both trimerise into six-bladed propellers with two blades per subunit. In fact the largest propeller of known structure, Ph1500 of Pyrococcus horikoshii, is a homo-hexamer with 12 blades in total (PDB:2M3X and 4UZR). Entire propellers can of course be duplicated in a single step. A WD40 propeller called WRAP from the nitrogen-fixing cyanobacterium Nostoc punctiforme has apparently arisen from a recent doubling to yield a polypeptide consisting of 14 nearly identical blades that fold into two seven-bladed propellers 


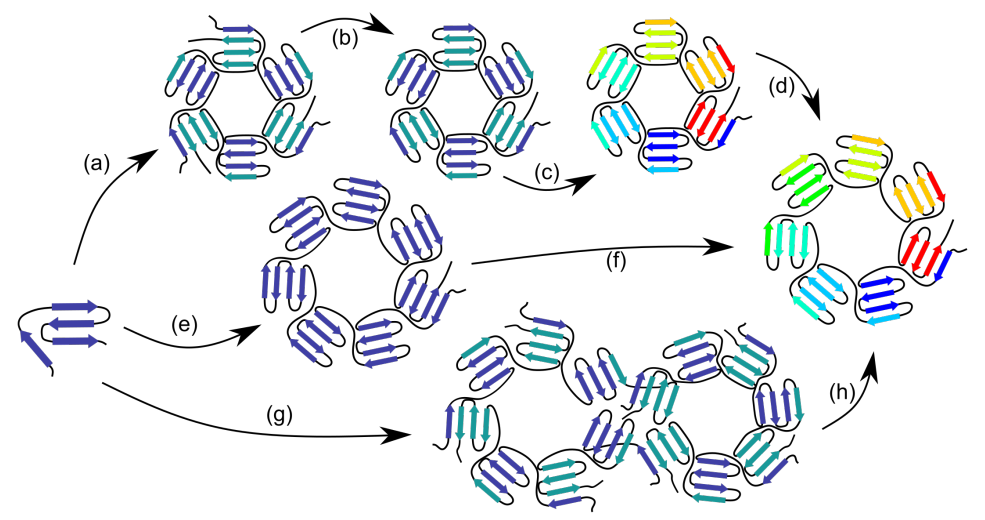

Figure 2: Possible pathways to $\beta$-propeller proteins. (a) A single fragment is duplicated and fused to create a multimeric even-bladed propeller. (b) Further duplication yields a symmetric monomer which subsequently (c) diversifies under evolutionary pressure. (d) Structural plasticity allows a single blade deletion or insertion to make an odd-numbered propeller. (e) Direct multiple duplication to an oddbladed monomer which (f) later diversifies. (g) Multiple multimeric domain-swapped intermediates arise that $(\mathrm{h})$ further evolve into a single propeller.

(PDB:2YMU) [18]. This organism has a notable number of propellers with high internal symmetry, indicating recent appearance [14]. Prokaryotic WD propeller proteins are relatively rare, and show a greater tendency to strong internal sequence conservation than eukaryotic propellers [19], which has led to speculation that the gene family arose in eukaryotes and subsequently transferred to bacteria [20].

As repeat proteins, $\beta$-propellers provide us with both analytic and synthetic approaches to understanding their evolutionary origins. In a recent paper, for example, Lupas and colleagues used WRAP to show that $\beta$-propellers built from identical repeat sequences can show remarkable structural plasticity [21]. They expressed tandem repeats of this blade with up to six copies per polypeptide. Two- and four-bladed fragments self-assemble into an eight-bladed propeller, while the three-bladed fragment on the other hand assembles as a trimer. None adopt the original seven-bladed fold. The assemblies possess a slightly distorted architecture, with the triple repeat becoming triangular and the quadruple repeat becoming oval. The five-bladed fragment dimerises asymmetrically; one subunit forms five normal blades, and the other subunit forms four blades and an $\alpha$-helix. The subunits associate to form a propellerlike structure, but with the blades of one subunit anti-parallel to those of the other. This variety of forms suggests that the natural seven-bladed structure is selected by kinetic processes during folding, rather than being hard-wired by the sequence. By breaking symmetry the protein can access an energy landscape whose minima represent very different structures. These multimers provide evidence that malleable $\beta$-propeller sequences could well have stabilised evolutionary intermediates between propellers with different numbers of blades (Figure 2).

Protein design has also proved to be a valuable alternative tool to investigate evolutionary pathways. A computational method based on ancestral sequence reconstruction was used to create Pizza protein, consisting of six identical blades, from an NHL-motif propeller template with a very regular structure [22]. Smaller fragments of Pizza with only two or three blades self-assembled into six-bladed oligomers. It was also shown that different numbers of repeats combine by domain-swapping so that, for example, a four-bladed repeat trimerised into two six-bladed propellers. Expressing the single repeat alone however did not give a folded protein. This method was repeated with the eight-bladed human F-box protein, a WD40 propeller. The first design, called Tako, formed a stable propeller with eight identical blades but was unable to assemble from smaller fragments. A second, four-fold symmetrical protein called Ika was therefore designed using Toulbar2, an AI function minimizer [23, 24]. The repeat sequence of Ika forms two blades that carry complementary surface charges. Ika is more stable than Tako, and can produce oligomeric forms, suggesting that loosening symmetry restraints can assist folding [25].

Some years ago the group of Tawfik screened libraries of fragments, about 100 residues long, of the five-bladed propeller tachylectin-2 for folding and self-assembly [26]. They found sequences that were able to multimerise as asymmetrically domain-swapped propellers, close to tachylectin- 2 in shape, connected by either one or two linkers. The subunits show remarkable plasticity to adopt the $\beta$-propeller form, which 
may partly explain the evolutionary success of the superfamily. Propellers built from double repeats could even be evolutionary intermediates en route to proteins with an odd number of blades. In more recent research that combined directed evolution with ancestral sequence reconstruction, the same group found a single repeat that can multimerise into the five-bladed propeller. This is currently the only $\beta$-propeller known that can self-assemble from a single blade, and it is striking how much experimental effort was required to discover it [27]. Natural evolution possibly involves an early phase with single blades attached to other sequences that assist the folding process.

Pizza, Tako and the tachylectin-2-based designs show a strong preference for the symmetry of their natural template proteins, regardless of how many blades are present on the subunit. This behaviour is typical of other closed repeat proteins such as the $\beta$-trefoil proteins, which invariably show intrinsic three-fold symmetry. A symmetrical artificial trefoil, symfoil-4P, also used domain-swapping to preserve its natural structure when expressed as a double tandem repeat [28]. $\beta$-propellers are unusual in that the number of repeats varies widely throughout the family, suggesting this fold is flexible enough to evolve through jumps in blade number. Furthermore, some propellers with different symmetry show closelyrelated sequences, hinting that such changes have occurred relatively late in the evolutionary process.

Protein design has provided further support for the role of structural plasticity in propeller evolution (Figure 3). Ancestral reconstruction was applied to the unpublished structure of an incomplete $\beta$-propeller apparently missing one or more blades (PDB:3HXJ), yielding a repeat sequence called Cake. Linking nine copies of Cake into one polypeptide yielded Cake9, a stable and perfectly symmetrical nine-bladed propeller [29]. Expressing different repeat lengths allowed four different architectures to be identified. Cake3 also made a nine-bladed propeller. The two-, five- and ten-bladed fragments fold into a ninebladed propeller with one disordered blade, while the four- and eight-bladed fragments make symmetrical eight-bladed propellers. Cake6 formed a trimer folded into two domain-swapped nine-bladed propellers reminiscent of the domain-swapped tachylectin-2. Cake7 formed a larger multimer but its structure could not be determined. The Cake blade showed no evidence of any strain in crystal structures of the eight- and nine-bladed propellers, suggesting that changes in repeat number by one can occur, given a sufficiently forgiving sequence [29]. A similar symmetry change was demonstrated in the cyclic protein TRAP many years ago [30].

\subsection{Closure mechanism}

Most of the studies discussed above focused on the variability of the repeat number, but another important aspect of $\beta$-propellers is the "velcro" closure, which results from a frame-shift between the structural blade and the sequence repeat. Other closed repeat proteins such as $\beta$-trefoils also show a similar characteristic property [31]. Almost all propellers have such a feature, apart from some four-bladed propellers that use a disulphide bond to link the chain termini [32]. A notable exception is the sub-family of prolyloligopeptidases in which the lack of "velcro" straps enhances their catalytic activity [33]. The location of the polypeptide termini within the blade differs between families. The four $\beta$-strands of one blade are connected by three loops, and the chain termini may be found at any of these loop regions. Both the inside position (between the two strands closest to the central channel), and the outside position (between the two outermost strands) are commonly found in nature. The middle position has only been observed in 'Regulator of chromosome condensation'-like domains [34], a family that was found to lie outside the main $\beta$-propeller cluster in a recent bio-informatics study [35].

In order to investigate the differences between the different "velcro" positions, cyclic permutants were made of the Pizza protein, which can serve as a useful model system because of its symmetrical nature [36]. The mutants with no "velcro" folded but showed lower stability. Even though the natural template for Pizza has an outside "velcro", moving the chain termini to the inside position improved resistance to heat and chemical denaturation. Only the outside "velcro" allowed the protein to self-assemble from smaller fragments, however, suggesting that the position of the chain termini may reflect the evolutionary path via oligomeric intermediates. This contrasts with the findings from the development of novel tachylectin2-based propellers. Attempts to recapitulate the evolution of this five-bladed propeller from frame-shifted repeats proved unsuccessful, and non-velcro fragments had to be used [27]. For tachylectin-2, therefore, the velcro may have arisen later in evolution. $\beta$-propeller families show considerable variation in their exon arrangements, also suggesting that there is no strongly favoured trajectory for their evolution from 

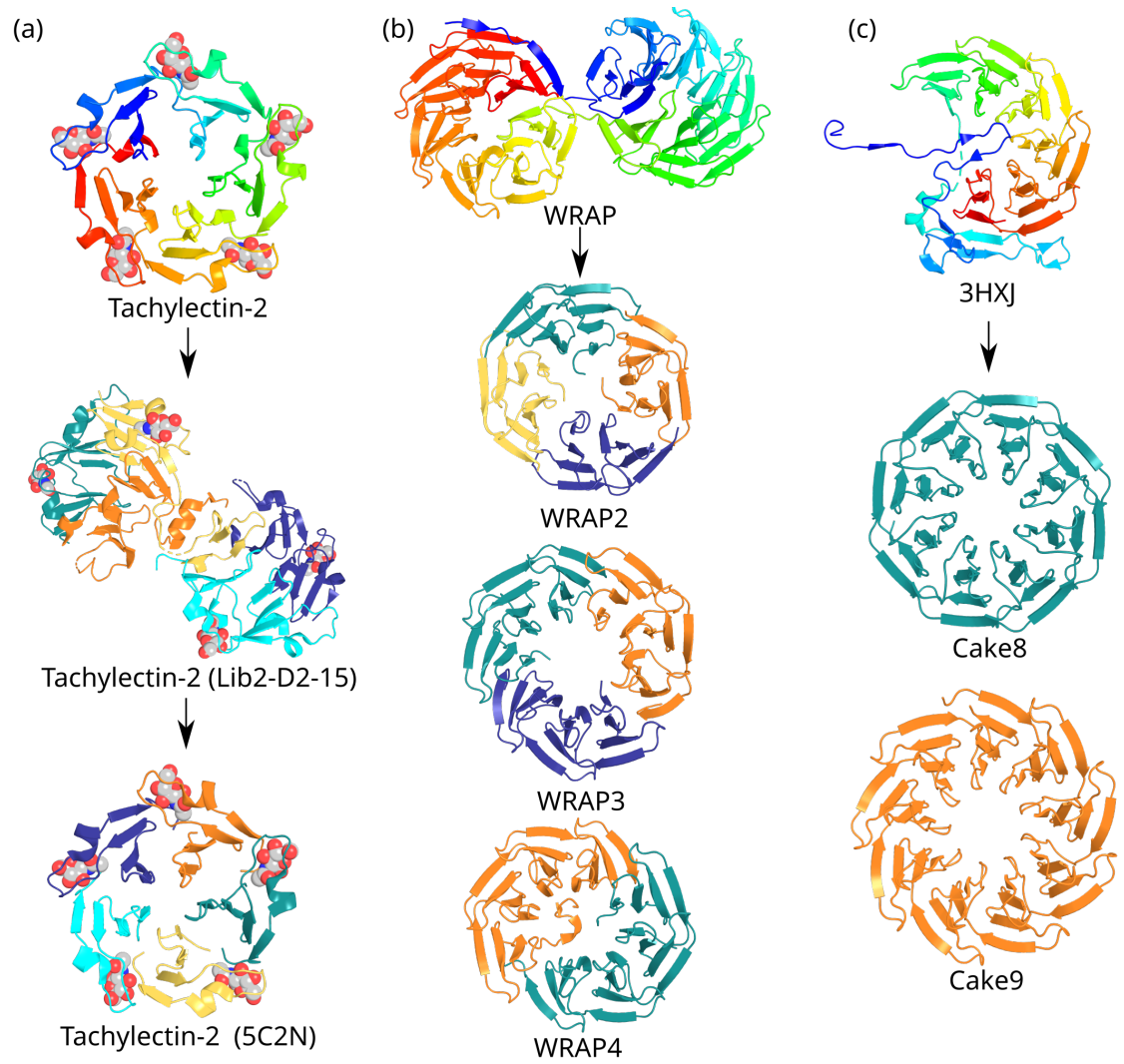

Figure 3: Symmetry making and breaking in artificial constructs. (a) Two-bladed fragments from a library based on tachylectin-2 adopt a domain-swapped conformation. Repeated library construction and directed evolution steps eventually led to a single blade that assembled into a homo-pentameric propeller. (b) The natural 14-bladed protein template for the WRAP motif. Double or quadruple repeats fold into distorted eight-bladed propellers. The triple repeat forms a nine-bladed propeller (PDB:6R5Z). (c) An incomplete $\beta$-propeller from M. maripaludis was used as the template for the Cake motif, which adopts a symmetrical eight- or nine-bladed fold depending on the number of repeats expressed. 
primordial blade sequences [13].

\subsection{Oligomerization}

Some $\beta$-propellers assemble beyond simple, closed circular structures and adopt higher levels of association. These multimeric forms have mostly been observed for lectins, which are widely found to enhance avidity for their targets through multivalent interactions. The simplest are two-fold symmetrical dimers, sometimes stabilised by disulphide bonds [37, 38, 39, 40]. An $\alpha$-L-arabinofuranosidase from Thermothielavioides terrestris is reported to be a monomeric five-bladed propeller in solution, that can form a domain-swapped dimer in cristallo, or possibly merely at high concentration [41]. A tetrameric complex built from two dimers connected by disulphides was observed in PLL, a fucose-binding lectin from Photorhabdus luminescens [42]. PHL and PLL2 are seven-bladed PropLecs from other Photorhabdus species that form stacked, "bangle-like" dimers [39, 43]. In 2018 the group of Varrot reported the structure of tectonin 2, a fungal lectin whose protomer forms a six-bladed propeller with six equivalent binding sites for O-methylated sugars such as 2-O-methyl- $\alpha$-L-fucose. The propeller assembles into a tetramer with one protomer forming each face of a tetrahedron with 24 binding sites [44]. These lectins are either toxins or part of the defence mechanism of the organism, and believed to aggregate pathogens or disrupt their cell membranes.

Recently the group of Imberty has produced a comprehensive curated structural database of lectins [45]. In 2019, the group derived hidden Markov models (HMMs) for five different propeller lectin blades, and used these to predict almost 4000 lectins built from tandem repeats in unannotated genomic sequences [40]. The signature motifs extracted for five different groups (two of them six-bladed) are remarkably different. Evidence of polypeptides carrying three blades was found amongst these sequences, suggesting they dimerise into six-bladed propellers, a form that had not been found before. To round out this impressive tour de force, one such lectin, KozL from K. zhangzhouensis, was consequently expressed in E. coli, leading to a high-resolution crystal structure (PDB:6HTN) that confirmed the predicted dimeric structure [40]. It is not currently clear however why such homo-oligomeric propeller proteins should be so rare, as these forms are not necessarily unstable. Many lectins are found to be repeat proteins with one binding site per motif repeat, allowing the simultaneous binding of several targets. KozL for example has six fucose sites per molecule. These highly multivalent proteins are of interest not only from an evolutionary or functional perspective, but also as structures that can be engineered for medical and biotechnological purposes [9].

\section{Conclusion}

Although the intrinsically symmetrical structure of proteins such as $\beta$-propellers is obvious to the eye, it has taken numerous detailed studies to show that tandem repetition of identical copies of sequences can yield stable folds. In general, consensus methods alone do not provide sequence motifs that fold well, and transitions between symmetry states, for example by increasing blade number, are not trivial. With considerable effort, attempts to reproduce the evolution of toroidal folds from some $U r$-motif have yielded well-behaved and highly symmetrical artificial proteins. Surprisingly, some of the derived repeat sequences have shown a tendency to break symmetry, sometimes dramatically so, and protein design is a useful tool to investigate the limits to adaptation within a symmetry rule. Analysis of modern protein sequence space concurs with studies of individual proteins that motif reuse is fundamental to protein evolution [46]. Kleptomania is possibly as much in evidence as bricolage. It is still not clear though what causes some sequences to adapt flexibly to different architectures, and greater understanding of these fragments may well greatly shrink the immense volumes of sequence space to be searched in protein design. Recent progress in tailoring toroid proteins has been hugely successful however, and these proteins hold great promise as clinical and biotechnological tools. 


\section{Acknowledgements}

BM thanks Research Foundation Flanders for a fellowship (GBM-D3229-ASP/17). ARDV thanks Research Foundation Flanders for financial support (G0E4717N, G0F9316N and G051917N). JRHT thanks OpenEye Scientific Software for financial support.

\section{Declaration of Interests}

The authors declare no conflict of interest.

\section{References}

[1] Vikram Alva, Johannes Söding, and Andrei N Lupas. A vocabulary of ancient peptides at the origin of folded proteins. eLife, 4:e09410, dec 2015.

[2] Miguel A. Andrade, Carolina Perez-Iratxeta, and Chris P. Ponting. Protein repeats: Structures, functions, and evolution. J. Struct. Biol., 134(2):117-131, 2001.

[3] Matteo Delucchi, Elke Schaper, Oxana Sachenkova, Arne Elofsson, and Maria Anisimova. A new census of protein tandem repeats and their relationship with intrinsic disorder. Genes, 11(4):407, apr 2020 .

[4] Lisanna Paladin, Layla Hirsh, Damiano Piovesan, Miguel A. Andrade-Navarro, Andrey V. Kajava, and Silvio C.E. Tosatto. RepeatsDB 2.0: improved annotation, classification, search and visualization of repeat protein structures. Nucleic Acids Res., 45(D1):D308-D312, 112017.

[5] Junan Li, Anjali Mahajan, and Ming-Daw Tsai. Ankyrin repeat: A unique motif mediating proteinprotein interactions. Biochemistry, 45(51):15168-15178, 2006. PMID: 17176038.

[6] Reinhard Sterner and Birte Höcker. Catalytic versatility, stability, and evolution of the $(\beta \alpha)_{8}$-barrel enzyme fold. Chem. Rev., 105(11):4038-4055, 2005. PMID: 16277370.

[7] Shalini Iyer, Robert Visse, Hideaki Nagase, and K. Ravi Acharya. Crystal structure of an active form of human MMP-1. J. Mol. Biol., 362(1):78 - 88, 2006.

[8] Esben M Quistgaard, Peder Madsen, Morten K Grøftehauge, Poul Nissen, Claus M Petersen, and Søren S Thirup. Ligands bind to Sortilin in the tunnel of a ten-bladed $\beta$-propeller domain. Nat. Struct. Mol. Biol., 16(1):96-98, jan 2009.

[9] Simona Notova, François Bonnardel, Frédérique Lisacek, Annabelle Varrot, and Anne Imberty. Structure and engineering of tandem repeat lectins. Curr. Opin. Struct. Biol., 62:39-47, jun 2020.

[10] Cammy K.M. Chen, Nei Li Chan, and Andrew H.J. Wang. The many blades of the $\beta$-propeller proteins: Conserved but versatile. Trends in Biochemic Sci., 36(10):553-561, oct 2011.

[11] Vilmos Fülöp and David T Jones. $\beta$-Propellers: structural rigidity and functional diversity. Curr. Opin. Struct. Biol., 9:715-721, 1999.

[12] Vikram Alva and Andrei N Lupas. From ancestral peptides to designed proteins. Curr Opin Struct Biol., 48:103-109, feb 2018.

[13] Lisanna Paladin, Marco Necci, Damiano Piovesan, Pablo Mier, Miguel A. Andrade-Navarro, and Silvio C.E. Tosatto. A novel approach to investigate the evolution of structured tandem repeat protein families by exon duplication. J. Struc Biol., page 107608, September 2020.

[14] Indronil Chaudhuri, Johannes Söding, and Andrei N. Lupas. Evolution of the $\beta$-propeller fold. Proteins Struct. Funct. Bioinforma., 71(2):795-803, 2008. 
[15] Chao Xu and Jinrong Min. Structure and function of WD40 domain proteins. Protein E Cell, 2(3):202-214, March 2011.

[16] Nikola Kostlánová, Edward P. Mitchell, Hugues Lortat-Jacob, Stefan Oscarson, Martina Lahmann, Nechama Gilboa-Garber, Gérard Chambat, Michaela Wimmerová, and Anne Imberty. The fucosebinding lectin from Ralstonia solanacearum. J. Biol. Chem., 280(30):27839-27849, jul 2005.

[17] Aymeric Audfray, Julie Claudinon, Saïda Abounit, Nathalie Ruvoën-Clouet, Göran Larson, David F. Smith, Michaela Wimmerová, Jacques Le Pendu, Winfried Römer, Annabelle Varrot, and Anne Imberty. Fucose-binding lectin from opportunistic pathogen Burkholderia ambifaria binds to both plant and human oligosaccharidic epitopes. J. Biol. Chem., 287(6):4335-4347, feb 2012.

[18] Stanislaw Dunin-Horkawicz, Klaus O. Kopec, and Andrei N. Lupas. Prokaryotic ancestry of eukaryotic protein networks mediating innate immunity and apoptosis. J. Mol. Biol., 426(7):1568 - 1582, 2014.

[19] Xue-Jia Hu, Tuan Li, Yang Wang, Yao Xiong, Xian-Hui Wu, De-Lin Zhang, Zhi-Qiang Ye, and Yun-Dong Wu. Prokaryotic and highly-repetitive WD40 proteins: A systematic study. Scientific Reports, 7(1), September 2017.

[20] Temple F. Smith. Diversity of WD-Repeat proteins, pages 20-30. Springer New York, New York, NY, 2008.

[21] Evgenia Afanasieva, Indronil Chaudhuri, Jörg Martin, Eva Hertle, Astrid Ursinus, Vikram Alva, Marcus D. Hartmann, and Andrei N. Lupas. Structural diversity of oligomeric $\beta$-propellers with different numbers of identical blades. eLife, 8:e49853, oct 2019.

[22] Arnout R.D. Voet, Hiroki Noguchi, Christine Addy, David Simoncini, Daiki Terada, Satoru Unzai, Sam Yong Park, Kam Y.J. Zhang, and Jeremy R.H. Tame. Computational design of a self-assembling symmetrical $\beta$-propeller protein. Proc. Natl. Acad. Sci. U.S.A., 111(42):15102-15107, oct 2014.

[23] Barry Hurley, Barry O'Sullivan, David Allouche, George Katsirelos, Thomas Schiex, Matthias Zytnicki, and Simon de Givry. Multi-language evaluation of exact solvers in graphical model discrete optimization. Constraints, 21(3):413-434, jul 2016.

[24] David Simoncini, David Allouche, Simon de Givry, Céline Delmas, Sophie Barbe, and Thomas Schiex. Guaranteed Discrete Energy Optimization on Large Protein Design Problems. J. Chem. Theory Comput., 11(12):5980-5989, dec 2015.

[25] Hiroki Noguchi, Christine Addy, David Simoncini, Staf Wouters, Bram Mylemans, Luc Van Meervelt, Thomas Schiex, Kam Y.J. Zhang, Jeremy R.H. Tame, and Arnout R.D. Voet. Computational design of symmetrical eight-bladed $\beta$-propeller proteins. IUCrJ, 6(1):46-55, jan 2019.

[26] Itamar Yadid, Noam Kirshenbaum, Michal Sharon, Orly Dym, and Dan S. Tawfik. Metamorphic proteins mediate evolutionary transitions of structure. Proc. Natl. Acad. Sci. U.S.A., 107(16):7287$7292,2010$.

[27] Robert G. Smock, Itamar Yadid, Orly Dym, Jane Clarke, and Dan S. Tawfik. De Novo Evolutionary Emergence of a Symmetrical Protein Is Shaped by Folding Constraints. Cell, 164(3):476-486, jan 2016.

[28] Jihun Lee and Michael Blaber. Experimental support for the evolution of symmetric protein architecture from a simple peptide motif. Proc. Natl. Acad. Sci. U.S.A., 108(1):126-130, 2011.

[29] Bram Mylemans, Ina Laier, Kenichi Kamata, Satoko Akashi, Hiroki Noguchi, Jeremy R. H. Tame, and Arnout R. D. Voet. Structural plasticity of a designer protein sheds light on $\beta$-propeller protein evolution. FEBS J., page febs.15347, may 2020.

[30] Jonathan G. Heddle, Takeshi Yokoyama, Ichiro Yamashita, Sam-Yong Park, and Jeremy R.H. Tame. Rounding up: Engineering 12-membered rings from the cyclic 11-mer TRAP. Structure, 14(5):925933, May 2006.

[31] Connie A. Tenorio, Liam M. Longo, Joseph B. Parker, Jihun Lee, and Michael Blaber. Ab initio Folding of a Trefoil-fold Motif Reveals Structural similarity with a $\beta$-propeller Blade Motif. Protein Sci., 29(5):1172-1185, mar 2020. 
[32] J Li, P Brick, MC O’Hare, T Skarzynski, LF Lloyd, VA Curry, IM Clark, HF Bigg, BL Hazleman, TE Cawston, and DM Blow. Structure of full-length porcine synovial collagenase reveals a C-terminal domain containing a calcium-linked, four-bladed $\beta$-propeller. Structure, 3(6):541-549, jun 1995.

[33] Vilmos Fülöp, Zoltán Szeltner, and László Polgár. Catalysis of serine oligopeptidases is controlled by a gating filter mechanism. EMBO Rep., 1(3):277-281, sep 2000.

[34] Louis Renault, Nicolas Nassar, Ingrid Vetter, Jörg Becker, Christian Klebe, Michel Roth, and Alfred Wittinghofer. The $1.7 \AA$ crystal structure of the regulator of chromosome condensation (RCC1) reveals a seven-bladed propeller. Nature, 392(6671):97-101, mar 1998.

[35] Klaus O. Kopec and Andrei N. Lupas. $\beta$-Propeller Blades as Ancestral Peptides in Protein Evolution. PLoS ONE, 8(10), oct 2013.

[36] Bram Mylemans, Hiroki Noguchi, Els Deridder, Eveline Lescrinier, Jeremy R.H. Tame, and Arnout Voet. Influence of circular permutations on the structure and stability of a six-fold circular symmetric designer protein. Prot. Sci., X(X):1-2, December 2020.

[37] Michaela Wimmerova, Edward Mitchell, Jean-Frederic Sanchez, Catherine Gautier, and Anne Imberty. Crystal structure of fungal lectin. J. Biol. Chem, 278(29):27059-27067, May 2003.

[38] Josef Houser, Jan Komarek, Nikola Kostlanova, Gianluca Cioci, Annabelle Varrot, Sheena C. Kerr, Martina Lahmann, Viviane Balloy, John V. Fahy, Michel Chignard, Anne Imberty, and Michaela Wimmerova. A Soluble Fucose-Specific Lectin from Aspergillus fumigatus Conidia - Structure, Specificity and Possible Role in Fungal Pathogenicity. PLoS ONE, 8(12):e83077, dec 2013.

[39] Gita Jančaříková, Josef Houser, Pavel Dobeš, Gabriel Demo, Pavel Hyršl, and Michaela Wimmerová. Characterization of novel bangle lectin from Photorhabdus asymbiotica with dual sugar-binding specificity and its effect on host immunity. PLOS Pathog, 13(8):1-28, August 2017.

[40] François Bonnardel, Atul Kumar, Michaela Wimmerova, Martina Lahmann, Serge Perez, Annabelle Varrot, Frédérique Lisacek, and Anne Imberty. Architecture and Evolution of Blade Assembly in $\beta$-propeller Lectins. Structure, 27(5):764-775.e3, may 2019.

[41] Suelen Camargo, Evandro J. Mulinari, Leonardo R. de Almeida, Amanda Bernardes, Rolf A. Prade, Wanius Garcia, Fernando Segato, and João R.C. Muniz. Functional and structural characterization of an $\alpha$-L-arabinofuranosidase from Thermothielavioides terrestris and its exquisite domain-swapped $\beta$ propeller fold crystal packing. Biochim Biophys Acta Proteins Proteom, 1868(12):140533, December 2020.

[42] Atul Kumar, Petra Sýkorová, Gabriel Demo, Pavel Dobeš, Pavel Hyršl, and Michaela Wimmerová. A novel fucose-binding lectin from Photorhabdus luminescens (PLL) with an unusual heptabladed $\beta$-propeller tetrameric structure. J. Biol. Chem., 291(48):25032-25049, nov 2016.

[43] Eva Fujdiarová, Josef Houser, Pavel Dobeš, Gita Paulíková, Nikolay Kondakov, Leonid Kononov, Pavel Hyršl, and Michaela Wimmerová. Heptabladed $\beta$-propeller lectins PLL2 and PHL from Photorhabdus spp. recognize O-methylated sugars and influence the host immune system. FEBS J., July 2020 .

[44] Roman Sommer, Olga N. Makshakova, Therese Wohlschlager, Stephanie Hutin, May Marsh, Alexander Titz, Markus Künzler, and Annabelle Varrot. Crystal Structures of Fungal Tectonin in Complex with O-Methylated Glycans Suggest Key Role in Innate Immune Defense. Structure, 26(3):391402.e4, mar 2018.

[45] François Bonnardel, Julien Mariethoz, Sebastian Salentin, Xavier Robin, Michael Schroeder, Serge Perez, Frédérique Lisacek, and Anne Imberty. UniLectin3D, a database of carbohydrate binding proteins with curated information on 3D structures and interacting ligands. Nucleic Acids Res., 47(D1):D1236-D1244, January 2019.

[46] Noelia Ferruz, Francisco Lobos, Dominik Lemm, Saacnicteh Toledo-Patino, José Arcadio FaríasRico, Steffen Schmidt, and Birte Höcker. Identification and Analysis of Natural Building Blocks for Evolution-Guided Fragment-Based Protein Design. J. Mol. Biol., 432(13):3898-3914, jun 2020. 